\title{
SHAPE IDENTIFICATION IN NONLINEAR BOUNDARY PROBLEMS SOLVED BY PIES METHOD
}

\author{
Eugeniusz ZIENIUK*, Agnieszka BOŁTUĆ', Krzysztof SZERSZEŃ*
}

\author{
*Faculty of Mathematics and Informatics, University of Bialystok, ul. Sosnowa 64, 15-887 Bialystok, Poland
}

ezieniuk@ii.uwb.edu.pl, aBołtuć@ii.uwb.edu.pl, kszerszen@ii.uwb.edu.pl

\begin{abstract}
The paper presents the strategy for identifying the shape of defects in the domain defined in the boundary value problem modelled by the nonlinear differential equation. To solve the nonlinear problem in the iterative process the PIES method and its advantages were used: the efficient way of the boundary and the domain modelling and global integration. The identification was performed using the genetic algorithm, where in connection with the efficiency of PIES we identify the small number of data required to the defect's definition. The strategy has been tested for different shapes of defects.
\end{abstract}

Key words: PIES, Defect, Shape Identification, Nonlinear Equation

\section{INTRODUCTION}

Identification of the shape of the boundary is classified as inverse problem, which next to direct issues are one of the two main groups of boundary value problems. Inverse boundary problems include problems related to the identification of body parameters, boundary conditions, shape or wide class of optimization problems (Tikhonov and Arsenin, 1977). These problems are described as ill-conditioned and characterized by difficulty in obtaining unequivocal solutions. There is the whole range of methods (Liu and Han, 2003) used to solve problems of this type, but the most popular are methods based on the minimization of the adopted objective function. In practice, this leads to iterative solving of the direct problem with the modified shape of the boundary. The problem, therefore, is to select a suitable method for numerical solving of the direct problem, and only then to assume a method for identification.

The numerical solution of the direct problem can be obtained by popular FEM (Zienkiewicz, 1977) and BEM (Ameen, 2001; Cholewa et al., 2002; Ligget and Salmon, 1981). In the case of BEM to define the boundary geometry are used various shape functions i.a. polynomials (Ameen, 2001) or their modification as cubic splines (Durdola and Fenner, 1990; Ligget and Salmon, 1981; Sen, 1995) or many others. These methods, regardless of applied shape functions, do not meet the requirement of the simplicity of defining and modifying the domain and the boundary, which is particularly important in the case of the problems of shape optimization or identification (Cerrolaza et al., 2000; Rus and Gallego, 2002). Thus, in FEM and BEM in each step of the iterative process, a re-discretization of the modified shape is required. Such conditioning of mentioned methods can cause a rapid increase in the number of design variables identified with nodes of the element mesh, regardless of shape functions applied for its definition.

Considering identification problems solved by BEM, in the literature there are attempts to the approximation of the identified part of the boundary in a parametric way (by Bézier or B-spline curves (Cholewa et al., 2002; Nowak et al., 2002). Such approximation of the boundary makes it easy to modify, but solution of the problem using BEM still requires its division into boundary elements. In order to obtain solutions with high accuracy it is necessary to divide even short identified part of the boundary into quite a lot of boundary elements.

Taking into account mentioned above problems, in the research (Zieniuk and Bołtuć, 2006; Zieniuk, 2007) carried out by authors the different approach to direct boundary problems has been proposed. Parametric integral equations system (PIES) has been obtained on the basis of the classic boundary integral equation (BIE) by its analytical modification (Zieniuk, 2007). That modification is concerned with the elimination of discretization both the domain (as in BEM) and the boundary by including the boundary geometry (defined in a parametric way by curves) directly in PIES kernels. In the case of solving nonlinear problems considered in the paper we should also define the domain of interest in order to made the integration over it (Zieniuk and Bołtuć, 2010). In BEM (where discretization of the domain was eliminated for problems without domain integrals), this requires dividing the domain into subdomains called cells. In the PIES method the domain, as the boundary, is not discretized, only created globally using surface patches. Modelling both the boundary by curves and the domain by surface patches requires a small number of control points, regardless of the complexity of the shape which is described by that curve or surface. The modification of such defined domain is very simple and effective with respect to the number of data. Advantages of such modification seem to be particularly important in the case of inverse tasks possible to solve by the repeatedly solving direct problem with the modified shape. Even more substantial is the fact, that in nonlinear problems modification of the shape at each iteration concerns not only the boundary, but also the domain. In the case of element methods such modification involves the division of the boundary and the domain into elements at each step of the algorithm, regardless of shape functions applied to their definition. In the PIES method the shape of the boundary and the domain is directly included into the mathematical formalism, so the modification of that shape will automatically change the PIES formalism, and its numerical solution does not require dividing such defined boundary into boundary elements, as is done in classical BIE. This approach requires the minimum set of data needed for modelling and modifying the shape of the boundary and the domain seems to be especially effective 
for shape identification and optimization problems.

The aim of this study is to apply the PIES method to identify the shape of defects in 2D boundary problems modelled by the nonlinear differential equation. The process of identification is performed iteratively, and at its each step the direct boundary value problem is solved by PIES. The complexity of the problem is that in the nonlinear boundary issues the direct problem is also solved in the iterative process. The identification process is steered by the genetic algorithm (Goldberg, 1989; Michalewicz, 1996), and the proposed strategy has been tested on examples with various shapes and number of defects.

\section{PIES FOR NONLINEAR BOUNDARY PROBLEMS}

In the presented paper the following partial differential equation (Zhu et al., 1998) is solved:

$\nabla u(\boldsymbol{x})+\varepsilon u(\boldsymbol{x})^{n}=p(\boldsymbol{x}), \boldsymbol{x} \in \Omega, n>1$

where: $\nabla^{2}=\frac{\partial^{2}}{\partial x_{1}^{2}}+\frac{\partial^{2}}{\partial x_{2}^{2}}$ is Laplace's operator, $p(\boldsymbol{x})$ is a given source function, $\varepsilon$ is a constant, and the domain $\Omega$ is enclosed by $\Gamma=\Gamma_{u} \cup \Gamma_{p}$ with boundary conditions $u=\bar{u}$ on $\Gamma_{u}$ and $\frac{\partial u}{\partial n} \equiv u=\bar{u}$ on $\Gamma_{p}$.

The form of PIES for considered in the paper equation is following (Zieniuk and Bołtuć, 2010):

$$
\begin{aligned}
0.5 u_{l}\left(s_{1}\right)=\sum_{j=1}^{n} \int_{s_{j-1}}^{s_{j}}\left\{\overline{\boldsymbol{U}}_{l j}^{*}\left(s_{1}, s\right) p_{j}(s)\right. \\
\\
\left.\quad-\overline{\boldsymbol{P}}_{l j}^{*}\left(s_{1}, s\right) u_{j}(s)\right\} J_{j}(s) d s
\end{aligned}
$$

$+\int_{\Omega} \overline{\overline{\boldsymbol{U}}}_{l}^{*}\left(s_{1}, y\right)\left[\varepsilon u(\boldsymbol{y})^{n}-p(\boldsymbol{y})\right] d \Omega(\mathrm{y})$

where: $J_{j}(s)=\left[\left(\frac{\partial \Gamma_{j}^{(1)}(s)}{\partial s}\right)^{2}+\left(\frac{\partial \Gamma_{j}^{(2)}(s)}{\partial s}\right)^{2}\right]^{0.5}, \quad s_{l-1} \leq s_{1} \leq$ $s_{l}, s_{j-1} \leq s_{1} \leq s_{j}, l=1,2, \ldots, n$ and $n$ is the number of segments .

The first and second integrands appering in (2) are respectively fundamental and singular solutions for Laplace's equation (they are presented in an explicit form in Zieniuk (2007).

Function $\overline{\boldsymbol{U}}_{l}^{*}$ from the domain integral from (2) takes the following form:

$\overline{\overline{\boldsymbol{U}}}_{l}^{*}\left(s_{1}, y\right)=\frac{1}{2 \pi} \ln \frac{1}{\left[\bar{\eta}_{1}^{2}+\bar{\eta}_{2}^{2}\right]^{0.5}}$

where $\bar{\eta}_{1}=\Gamma_{1}^{(1)}\left(s_{1}\right)-y_{1}, \bar{\eta}_{2}=\Gamma_{1}^{(2)}\left(s_{1}\right)-y_{2}$ whilst $\Gamma(\mathrm{s})$ are parametric curves which describe the boundary (in the paper Bézier curves of the first and third degree are used (Farin, 1990; Foley et al., 1994).

In order to obtain values of $u(\boldsymbol{x})$ in the domain $\Omega$ we have to use the integral identity, which can be expressed as follows:

$$
\begin{aligned}
u(\boldsymbol{x})=\sum_{j=1}^{n} \int_{s_{j-1}}^{s_{j}}\left\{\overline{\boldsymbol{U}}_{l j}^{*}(\boldsymbol{x}, s) p_{j}(s)\right. \\
\left.-\overline{\boldsymbol{P}}_{l j}^{*}(\boldsymbol{x}, s) u_{j}(s)\right\} J_{j}(s) d s
\end{aligned}
$$

$+\int_{\Omega} \widehat{\overline{\boldsymbol{U}}}_{l}^{*}(\boldsymbol{x}, \boldsymbol{y})\left[\varepsilon u(\boldsymbol{y})^{n}-p(\boldsymbol{y})\right] d \Omega(\mathrm{y})$ where: function $\widehat{\overline{\boldsymbol{U}}}_{l}^{*}$ from the integral over the domain takes the following form:

$\widehat{\overline{\boldsymbol{U}}}_{l}^{*}(\boldsymbol{x}, \boldsymbol{y})=\frac{1}{2 \pi} \ln \frac{1}{\left[\widehat{\eta}_{1}^{2}+\widehat{\eta}_{2}^{2}\right]^{0.5}}$

where $\hat{\bar{\eta}}_{1}=y_{1}-x_{1}, \hat{\bar{\eta}}_{2}=y_{2}-x_{2}, x_{1}, x_{2} \epsilon \Omega$.

The domain $\Omega$ is modelled using surface patches known from computer graphics (Farin, 1990; Foley et al., 1994). In the paper Bézier surfaces of the first and third degree were used.

\section{MODELING AND NUMERICAL SOLUTION}

The solution of PIES (2) is reduced to finding unknown functions $u_{j}(s)$ or $p_{j}(s)$ on each boundary segment of the considered problem. Unknown functions are approximated by expressions presented with details in Zieniuk (2007).

The equation (2) requires special treatment, because the integral over the domain contains a nonlinear and unknown at the same time function $u(\boldsymbol{x})^{n}$. For that reason, the application of the iterative process is necessary.

\subsection{Iterative process}

The equation (2) written down in the form of the algebraic equations system (after application the collocation method) can be presented as follows:

$$
\boldsymbol{H u}=\boldsymbol{G} \boldsymbol{p}+\boldsymbol{W}
$$

where: $\boldsymbol{H}=[H]_{M \times M}, \quad \boldsymbol{G}=[G]_{M \times M} \quad M=n \times N, N$ is the number of collocation points on the segment) are square matrices of elements expressed by integrals over the boundary from (2), $\boldsymbol{u}$ and $\boldsymbol{p}$ are vectors which contain the coefficients of approximation series (Zieniuk, 2007), whilst $\boldsymbol{W}$ is the vector of elements expressed by the integral over the domain from equation (2).

After application of boundary conditions and some transformations, the equation (6) takes the following form:

$\boldsymbol{A X}=\boldsymbol{F}+\boldsymbol{W}$

where: the vector $\boldsymbol{X}$ contains unknown coefficients of searched boundary approximating functions, whilst the vector $\boldsymbol{F}$ is known and depends on given boundary conditions.

The only problem is that the right-hand side $(\boldsymbol{W})$ of the equation (7) is unknown. It depends on the current value of solution $u_{i}(\boldsymbol{x})$ at chosen points of the domain $\Omega$. For that reason it is necessary to apply the iterative process and assume initial guess for searched solution $u_{0}(\boldsymbol{x})$. Taking into account the convergence of a method, most effective is to assume for the iteration $i=0$ real value of unknown function. It is also acceptable to choose constant or zero values.

After calculation $u_{1}(\boldsymbol{x})$ on the basis of (7), the solution becomes approximated in following iteration steps until fulfilling given stop criterion. The iterative process should be recognized as finished, if the difference between two lastly obtained values at all considered points of the domain (or the boundary) is smaller than the convergence criterion $\delta\left(\left|u_{i+1}(\boldsymbol{x})-u_{1}(\boldsymbol{x})\right| \leq \delta\right)$.

The proposed method of solving nonlinear boundary value problems has been tested taking into account the different shapes of domains, different boundary conditions and the degree of nonlinearity $(n=1,2,3)$ such as in Zieniuk and Boltuć (2010). The 
strategy has generated promising results: exact solutions in the rapidly converging iterative process.

\subsection{Modelling of the boundary and the domain}

A very important problem in identification problems is to effectively define the shape. Efficiency refers to the simplicity of both the modelling and modification. The PIES method is characterized by the replacement of modelling by elements with a more global modelling using parametric curves. In the paper, for modelling of the boundary we use Bézier curves of the first and third degree (Farin, 1990; Foley et al., 1994). They are characterized by the following advantages, which are crucial in identification process: easy representation of any shape using a small number of curves, a small number of data defining the shape of the curve and the ease of modification. The way of the definition of the boundary geometry in PIES using Bézier curves is presented in detail, inter alia, in Zieniuk (2007) and schematically in Fig. 1a,b.

a)

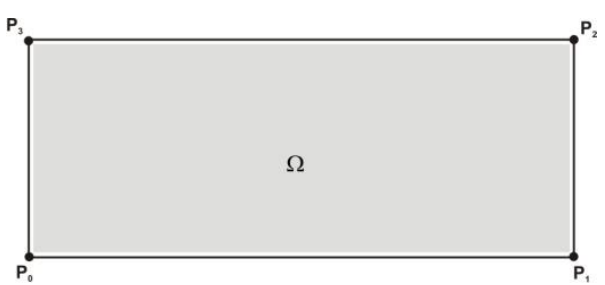

b)

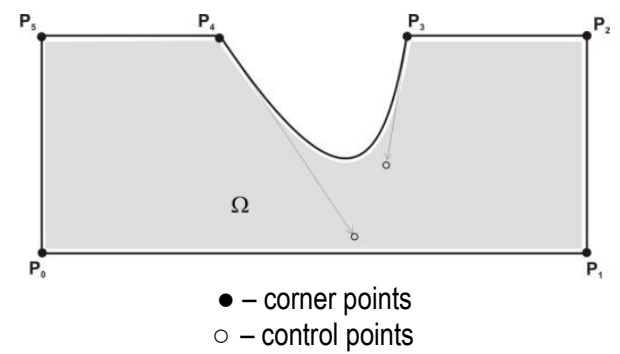

Fig. 1. Modelling of the boundary by curves of: a) the first, b) the first and third degree

Another problem related to modelling in nonlinear issues is to define the domain. In element methods it leads to discretization by finite elements in FEM or so-called cells in BEM. In PIES the domain is described globally using surface patches. Depending on the shape of the domain we use rectangular or triangular surfaces (Farin, 1990; Foley et al., 1994). The study takes into account rectangular surfaces: bilinear (for polygons) and bicubic (for curved boundary domains) (Farin, 1990; Foley et al., 1994). Important in such cases is also that defined in the 3D space surfaces are reduced to flat by eliminating the third dimension. The example of the modelling of the rectangular domain with one bilinear surface is presented in Fig. 1a. Details on the application of the various types of surface patches in the PIES method can be found, inter alia, in Bołtuć and Zieniuk (2011a, b).

In cases where we are dealing with more complex shapes, where the domain can not be modelled by one surface is required to use many of them. Fig. 2 presents the domain formed by two bilinear and one bicubic surface patches. This should not be identified with discretization well-known from element methods, where it is used not because of accurate description of the shape (as in the PIES method), but because of the accurate approximation of results.

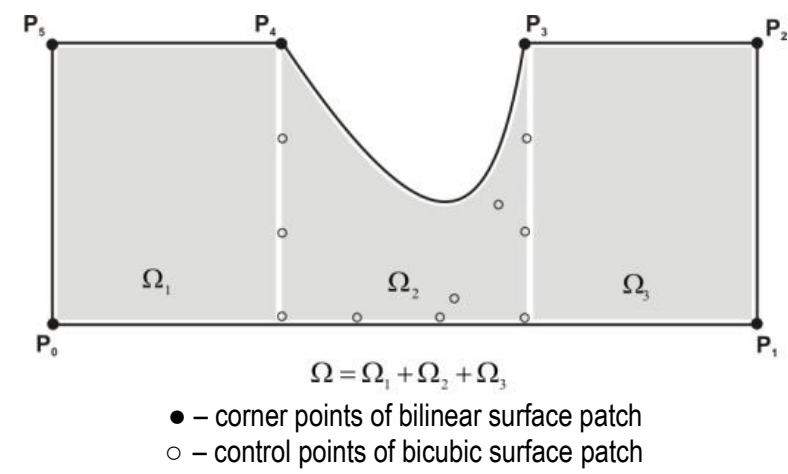

Fig. 2. Modelling of the domain by surface patches

In the case of the identification problem, at each step of the iterative process the direct boundary value problem is solved with the modified shape. When dealing with linear problems (or other that do not require integration over the domain) shape modification concerns only the boundary. Considering the geometry from Fig. 2, it would be steering by two control points that define the shape of the cubic Bézier curve mapping the part of the rectangle side. However, taking into account nonlinear problems (or other in which we deal with integrals over the domain), besides the boundary modified has to be also the shape of the surface patch, which define the modified domain. Thus, it is very important to apply modelling with reduced amount of data for modelling and modification. In the case of the PIES method and the geometry from Fig. 2, modification of the shape of the domain requires changing positions of only two control points which describe the surface corresponding to the domain $\Omega_{2}$. The other two bilinear patches do not change their shapes.

The integration over the domain in PIES is performed globally. In BEM integrals are calculated over subdomains called cells with the small number of weighting factors from the integration quadrature. The domain in PIES is defined globally by the small number of surfaces. The number of them depends only on the complexity of the shape of the modelled domain. Integrals are then calculated over the domain defined by the surface with a large number of quadrature points and optionally are aggregated when definition of the domain requires few surface patches. More on global integration over the domain in PIES can be found, among others in Bołtuć and Zieniuk (2011a, b) and Zieniuk and Bołtuć (2010).

\section{GA ALGORITHM}

Solved in the paper task of the shape identification is reduced to repeatedly solve the direct problem with the modified shape. The adopted approach generates many alternative solutions to the problem, among which we should indicate searched one taking into account introduced evaluation criterions. Because of the necessity of searching the large solution space we choose the genetic algorithm (GA) (Goldberg, 1989; Michalewicz, 1996) to control the process of identification. The success of the identification process is therefore dependent on the ability to seek appropriate solutions by GA. All those characteristics which distinguish AG from classical algorithms are favourable in the considered problem: the flexibility to choose the form of solutions, the 
parallel nature of the calculations or introducing an element of randomness and orientation of the searching process on the basis of the quality of actual solutions. Also relevant is the fact that GA are significantly resistant to existence of local minima in the search area.

Adjusting GA to the considered problem we should define the form of the chromosome and the fitness function. The shape is modified by means of control points $\mathrm{I}_{\mathrm{i}}\left(\mathrm{x}_{1 \mathrm{i}}, \mathrm{x}_{2 \mathrm{i}}\right)$ which define curves and surfaces, thus solving the problem is reduced to finding coordinates of $\mathrm{n}$ points (vector $\mathrm{X}$ ) that create the identified shape. Mentioned points are encoded in the GA chromosome as follows:

chromosome

$=\left\langle\mathrm{I}_{0}\left(\mathrm{x}_{10}, \mathrm{x}_{20}\right), \mathrm{I}_{1}\left(\mathrm{x}_{11}, \mathrm{x}_{21}\right), \ldots, \mathrm{I}_{\mathrm{n}}\left(\mathrm{x}_{1 \mathrm{n}}, \mathrm{x}_{2 \mathrm{n}}\right)\right\rangle$

Identification using PIES is reduced to finding such points determining the shape (vector $\mathrm{X}$ ), that solutions $u_{j}^{X}$ in $m$ selected measurement points are as close as possible to known reference values $u_{j}^{*}$. Such stated problem is the optimization task, with minimization of the following fitness function:

$$
f(X)=\sqrt{\sum_{j=1}^{m}\left(u_{j}^{X}-u_{j}^{*}\right)^{2}}
$$

We use algorithm bases on the classic Goldberg's scheme (Goldberg, 1989) and is implemented based on the objectoriented C++ library Galib (Wall, 1996).

\section{TESTING EXAMPLES}

In order to test the strategy for identifying the shape we consider the boundary problem defined by the nonlinear differential equation (1) of the following form:

$\nabla^{2} u(\boldsymbol{x})+\varepsilon u(\boldsymbol{x})^{2}=p(\boldsymbol{x}), \boldsymbol{x} \in \Omega$

The equation (10) was analyzed for two different shapes of the domain. Firstly we check the convergence and accuracy of results comparing with analytical solution

$u(\boldsymbol{x})=x_{1}^{2}+x_{2}^{2}$

with the form of the function $p(\boldsymbol{x})$

$p(\boldsymbol{x})=\varepsilon\left\{x_{1}^{2}+x_{2}^{2}\right\}^{2}+4$

The rest of parameters assumed for researches are: $\varepsilon=1$, constant initial value of the solution $u_{0}=18$ and the convergence criterion $\delta=0.0001$. The application of PIES to solving problems with nonlinearity have finished successfully (obtaining an accurate numerical solutions) and seems to be effective (an iterative process converges to a final solution in small number of steps).

The main subject considered in the paper is the identification of the shape of the unknown part of the boundary. Identification problem is formulated as follows: from the known values at the measurement points deployed in the domain and/or on the boundary and on the assumption that part of the boundary is known and does not change the shape we have searched unknown part. Due to the lack of empirical values at measurement points obtained from the experiment, a simulation was performed to yield these values on the basis of the solution of direct boundary problems with the assumed shape by PIES method. In view of the fact that measured data are always noisy we also made a simulation with some level of noise in the inputs.

Adopted GA parameters are: population size - 50, the number of generations - 60 , the mutation probability - $60 \%$, the crossover probability - $3 \%$ and 5 independent runs.

The first considered problem with posed boundary conditions is presented in Fig.3. The domain was modelled using three surfaces: one bicubic and two bilinear. There is one defect in the form of the indentation, which is identified by only two control points (०).

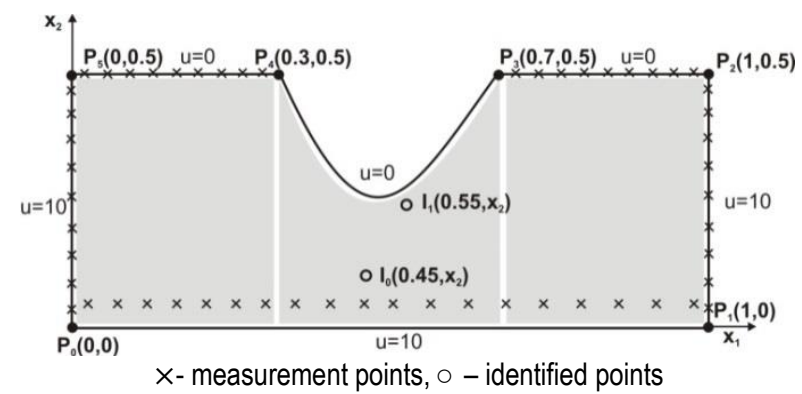

Fig. 3. Considered domain, measurement points and boundary conditions

Measurement points $(x)$ were considered taking into account three variants (illustratively shown in Fig.3):

a. forty five measurement points placed uniformly only in the domain at the horizontal cross-section $x_{2}=0.1$,

b. forty five measurement points placed only on the boundary,

c. ninety measurement points placed on the boundary and in the domain (the arrangement of points is taken from $a$ ) and $b$ )).

Only $x_{2}$ coordinates of two mentioned control points were identified in the search area corresponding to the height of the considered domain $0 \leq x_{2} \leq 0.5$. Average and the best results of identification for assumed coordinates $(0.1,0.4)$ are presented in Tab. 1.

As was mentioned above we also made a simulation with some level of noise in the inputs. The noise was generated randomly, and its maximum value was defined as $3 \%$ of the displacement at the measurement point. Tab. 1 also contains the results for distorted values at measurement points taking into account mentioned above three variants.

Values from Tab. 1 show the stability and high accuracy of the identification process even in examples with noise. It should be only noted that values of the fitness function in such cases increase.

Tab. 1. Identification results

\begin{tabular}{|c|c|c|c|}
\hline Variant & $\begin{array}{c}\text { avg fitness } \\
\text { function }\end{array}$ & $\begin{array}{c}\text { avg identified } \\
\text { value of } x_{2}\end{array}$ & $\begin{array}{c}\text { best identified } \\
\text { value of } x_{2}\end{array}$ \\
\hline a) without noise & 0.00458 & $\begin{array}{c}(0.09965, \\
0.40051)\end{array}$ & $\begin{array}{c}(0.09996, \\
0.40006)\end{array}$ \\
\hline b) without noise & 0.09894 & $\begin{array}{c}(0.10087, \\
0.39895)\end{array}$ & $\begin{array}{c}(0.09964, \\
0.40048)\end{array}$ \\
\hline c) without noise & 0.22518 & $\begin{array}{c}(0.103464, \\
0.395161)\end{array}$ & $\begin{array}{c}(0.100153, \\
0.399803)\end{array}$ \\
\hline a) with noise & 0.35488 & $\begin{array}{c}(0.100002, \\
0.400526\end{array}$ & $\begin{array}{c}(0.101616, \\
0.395862)\end{array}$ \\
\hline b) with noise & 1.97775 & $\begin{array}{c}(0.105792, \\
0.394575)\end{array}$ & $\begin{array}{c}(0.0981826, \\
0.411106)\end{array}$ \\
\hline c) with noise & 2.10746 & $\begin{array}{c}(0.094607, \\
0.406622)\end{array}$ & $\begin{array}{c}(0.099501, \\
0.401218)\end{array}$ \\
\hline
\end{tabular}


Fig.4 presents values of coordinates $x_{2}$ of identified points $\mathrm{I}_{0}$ and $\mathrm{I}_{1}$ obtained at following steps of GA (the case without noise, measurement points placed only in the domain). Presented values correspond to the best solutions in GA generations.

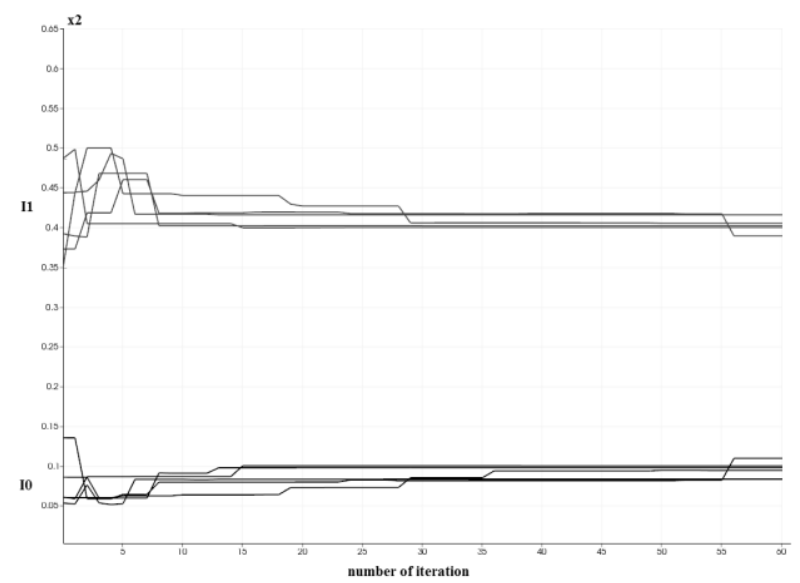

Fig. 4. Identified coordinates of two control points during iterative process

The shape of the second considered domain with posed boundary conditions is presented in Fig. 5 . As can be seen the domain is defined by four bilinear surface patches. There are two notches with symmetrical coordinate $x_{2}$ with respect to $x_{1}$ axis. Identification of the shapes of notches was performed using two corner points $I_{0}$ and $I_{1}$, but only three coordinates were searched (because of the $x_{2}$ symmetry). We have assumed nineteen measurement points placed uniformly at the horizontal crosssection in the middle of the considered polygon and fifty four measurement points on the boundary. Adopted GA parameters are the same as in the previous example.

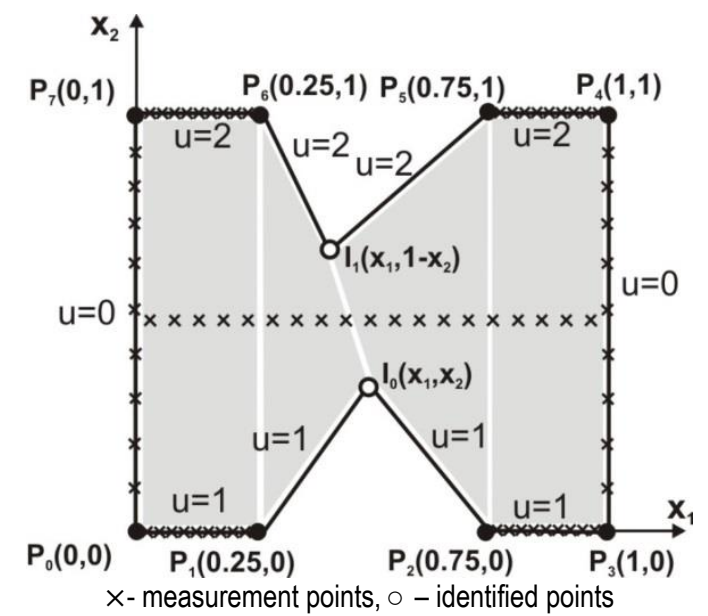

Fig. 5. Considered domain and measurement points

Assumed values of identified coordinates are following: $x_{1}$ of $I_{0}$ is $0.5625, x_{1}$ of $I_{1}$ is 0.4375 and $x_{2}$ is 0.8 . Tab. 2 presents the best identified points and values of the fitness function obtained after five independent runs of GA for measurement points placed in the domain only. The results for the case of measurement points located on the boundary are presented in Tab. 3. Tab. 4 and 5 present results of simulations with $3 \%$ level of noise in the measured data.
Tab. 2. Results of identification - measurement points in the domain

\begin{tabular}{|c|c|c|c|c|}
\hline \multirow{2}{*}{$\begin{array}{c}\text { number } \\
\text { of run }\end{array}$} & \multirow{2}{*}{$\begin{array}{c}\text { fitness } \\
\text { function }\end{array}$} & \multicolumn{3}{|c|}{ identified coordinates } \\
\cline { 3 - 5 } & & $x_{1}$ of $I_{0}$ & $x_{1}$ of $I_{1}$ & $x_{2}$ \\
\hline 1 & 0.00111 & 0.54577 & 0.44442 & 0.79973 \\
\hline 2 & $9.95 \mathrm{E}-06$ & 0.56248 & 0.43749 & 0.80000 \\
\hline 3 & 0.00034 & 0.56484 & 0.43643 & 0.79992 \\
\hline 4 & 0.00128 & 0.54444 & 0.44560 & 0.79964 \\
\hline 5 & 0.00086 & 0.55069 & 0.44201 & 0.79980 \\
\hline avg & $\mathbf{0 . 0 0 0 7 2}$ & $\mathbf{0 . 5 5 3 6 4}$ & $\mathbf{0 . 4 4 1 1 9}$ & $\mathbf{0 . 7 9 9 8 2}$ \\
\hline
\end{tabular}

Tab. 3. Results of identification - measurement points on the boundary

\begin{tabular}{|c|c|c|c|c|}
\hline \multirow{2}{*}{$\begin{array}{c}\text { number } \\
\text { of run }\end{array}$} & \multirow{2}{*}{$\begin{array}{c}\text { fitness } \\
\text { function }\end{array}$} & \multicolumn{3}{|c|}{ identified coordinates } \\
\cline { 3 - 5 } & & $x_{1}$ of $I_{0}$ & $x_{1}$ of $I_{1}$ & $x_{2}$ \\
\hline 1 & 0.00178 & 0.56249 & 0.43757 & 0.80005 \\
\hline 2 & 0.00079 & 0.56250 & 0.43753 & 0.80002 \\
\hline 3 & 0.02283 & 0.56283 & 0.43801 & 0.80079 \\
\hline 4 & 0.04477 & 0.56227 & 0.44154 & 0.79990 \\
\hline 5 & 0.00925 & 0.56275 & 0.43778 & 0.80031 \\
\hline avg & $\mathbf{0 . 0 1 5 8 8}$ & $\mathbf{0 . 5 6 2 5 7}$ & $\mathbf{0 . 4 3 8 4 9}$ & $\mathbf{0 . 8 0 0 2 2}$ \\
\hline
\end{tabular}

Tab. 4. Results of identification with noise - measurement points in the domain

\begin{tabular}{|c|c|c|c|c|}
\hline \multirow{2}{*}{$\begin{array}{c}\text { number } \\
\text { of run }\end{array}$} & \multirow{2}{*}{$\begin{array}{c}\text { fitness } \\
\text { function }\end{array}$} & \multicolumn{3}{|c|}{ identified coordinates } \\
\cline { 3 - 5 } & & $x_{1}$ of $I_{0}$ & $x_{1}$ of $I_{1}$ & $x_{2}$ \\
\hline 1 & 0.01374 & 0.52009 & 0.45675 & 0.79894 \\
\hline 2 & 0.01334 & 0.55750 & 0.44001 & 0.80038 \\
\hline 3 & 0.01256 & 0.60991 & 0.43188 & 0.80080 \\
\hline 4 & 0.01199 & 0.54149 & 0.45652 & 0.80041 \\
\hline 5 & 0.01342 & 0.60359 & 0.42998 & 0.80030 \\
\hline avg & $\mathbf{0 . 0 1 3 0 1}$ & $\mathbf{0 . 5 6 6 5 2}$ & $\mathbf{0 . 4 4 3 0 3}$ & $\mathbf{0 . 8 0 0 1 6}$ \\
\hline
\end{tabular}

Tab. 5. Results of identification with noise - measurement points on the boundary

\begin{tabular}{|c|c|c|c|c|}
\hline \multirow{2}{*}{$\begin{array}{c}\text { number } \\
\text { of run }\end{array}$} & \multirow{2}{*}{$\begin{array}{c}\text { fitness } \\
\text { function }\end{array}$} & \multicolumn{3}{|c|}{ identified coordinates } \\
\cline { 3 - 5 } & & $x_{1}$ of $I_{0}$ & $x_{1}$ of $I_{1}$ & $x_{2}$ \\
\hline 1 & 0.42872 & 0.59057 & 0.43730 & 0.80689 \\
\hline 2 & 0.41734 & 0.55781 & 0.43232 & 0.80011 \\
\hline 3 & 0.83691 & 0.55888 & 0.44005 & 0.79872 \\
\hline 4 & 0.44172 & 0.561135 & 0.442069 & 0.79598 \\
\hline 5 & 0.43116 & 0.551293 & 0.441299 & 0.799103 \\
\hline avg & $\mathbf{0 . 5 1 1 1 7 1}$ & $\mathbf{0 . 5 6 3 9 3 9}$ & $\mathbf{0 . 4 3 8 6 0 8}$ & $\mathbf{0 . 8 0 0 1 6 1}$ \\
\hline
\end{tabular}

As can be noticed from above tables, identified coordinates are very similar to assumed for all runs of GA. We have also considered the convergence of $\mathrm{GA}$, which is presented in the form of the fitness function for the best solution from each GA generation (the case with measurement points located on the boundary, without noise). The results for five independent runs of GA are given in Fig.6.

As can be seen in Fig. 6, the values of the fitness function in all considered runs of GA converges to zero, but the similar level of function values was obtained only for four runs after 20th generation. 


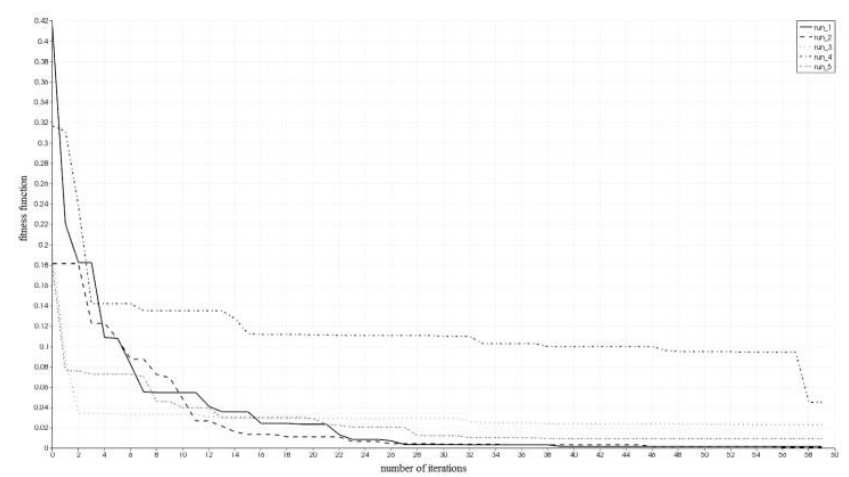

Fig. 6. Values of the fitness function during the iterative process for five independent runs of $G A$

It is also worth mentioning the convergence of the iterative process used to solve nonlinear boundary problems for each candidate solution of GA in each generation. The number of these iterations is $7-10$. Therefore, the number of independent solutions of boundary problems for the specific data and shape with assumed in the paper parameters of GA is approximately 2100030000 in one GA run. Such a large number of boundary problems to solve causes rising expectations for the method of its solution. Such a method should allows for the modelling of the shape with the minimum number of data, because it is modified each time. In addition, the system of equations solved in each case should be built with the least number of equations, in order to involve the least computer resources and minimize the computation time. These requirements are fulfilled by the proposed in the paper PIES method.

\section{CONCLUSIONS}

The paper presents the strategy for identifying the shape of the boundary defined in the nonlinear boundary problem. The concept has been applied and pre-verified on the example of the problem modelled by the nonlinear differential equation. The problem has been solved in the iterative process steering by GA and its complexity lies on the fact that to solve the nonlinear direct problem at each step of identification we have to also apply the iterative process. So we have to deal with the iterative process nested in the another iterative process.

The strategy proposed in the paper bases on PIES, which is characterized by that the changing position of control points causes changing the shape directly in PIES. In addition, PIES automatically adapts to the modified boundary, and the solution does not require division of the posed and approximated boundary into elements. An additional benefit is that the domain is modelled by surface patches, and integration over the domain is performed without division it into small cells as in BEM. It is done automatically for each modified by control points domain in the iterative process. Such opportunities do not have BEM at this stage of its development.

The algorithm has been tested on two examples with different shapes and with different defects, which were identified. Tests were successful - searched shapes have been identified, and both iterative processes have given satisfactory results in a small number of steps. These satisfactory results encourage further research and verification approach by applying it to problems characterized by a different source of nonlinearity e.g. nonlinear elasticity problems.

\section{REFERENCES}

1. Ameen M. (2001), Boundary element analysis. Theory and programming, Alpha Science International Ltd.

2. Aparicio N. D., Pidcock M. K. (1996), The boundary inverse problem for the Laplace equation in two dimensions, Inverse Problems, 12, 565-577.

3. Bołtuć A., Zieniuk E. (2011a), Modeling domains using Bézier surfaces in plane boundary problems defined by the Navier-Lame equation with body forces, Engineering Analysis with Boundary Elements, 35, 1116-1122.

4. Boltuć A., Zieniuk E. (2011b), PIES in problems of 2D elasticity with body forces on polygonal domains, J. Theor. Appl. Mech., 49/2, 369-384.

5. Cerrolaza M., Annicchiarico W., Martinez M. (2000), Optimization of $2 \mathrm{D}$ boundary element models using B-splines and genetic algorithms, Engineering Analysis with Boundary Elements, 24, 427-440.

6. Cholewa R., Nowak A. J., Bialecki R. A., Wrobel L. C. (2002), Cubic Bézier splines for BEM heat transfer analysis of the 2-D continuous casting problems, Computational Mechanics 28, 282-290.

7. Durodola J. F., Fenner R. T. (1990), Hermitian cubic boundary elements for two-dimensional potential problems, International Journal for Numerical Methods in Engineering, 30/5, 1051-1062.

8. Farin G. (1990), Curves and Surfaces for computer Aided Geometric Design, Academic Press Inc., San Diego.

9. Foley J., van Dam A., Feiner S., Hughes J., Phillips R. (1994), Introduction to Computer Graphics, Addison-Wesley.

10. Goldberg D.E. (1989), Genetic Algorithms in Search, Optimization and Machine Learning, Addison-Wesley Publishing Company, Massachusetts

11. Ligget J. A., Salmon J. R. (1981), Cubic spline boundary element International Journal for Numerical Methods in Engineering, 17, 543-556.

12. Liu G.R., Han X. (2003), Computational inverse techniques in nondestructive evaluation, CRC Press LLC.

13. Michalewicz Z. (1996), Genetic Algorithms + Data Structures = Evolution Programs, Springer-Verlag, Berlin.

14. Nowak I., Nowak A. J., Wrobel L. C. (2002) Identification of phase change front by Bézier splines and BEM, International Journal of Thermal Sciences, 41, 492-499.

15. Rus G., Gallego R. (2002), Optimization algorithms for identification inverse problems with the boundary element method, Engineering Analysis with Boundary Elements, 26, 315-327.

16. Sen D. (1995), A cubic-spline boundary integral method for twodimensional free-surface flow problems. International Journal for Numerical Methods in Engineering, 38/11, 1809-1830.

17. Tikhonov A.N., Arsenin V.Y. (1977), Solution of III-posed problems, John Wiley \& Sons, New York.

18. Wall M. (1996), GAlib: A C++ Library of Genetic Algorithm Components, version 2.4, Mechanical Engineering Department, Massachusetts Institute of Technology (http://lancet.mit.edu/ga/).

19. Zhu T., Zhang J., Atluri N. (1998), A meshless local boundary integral equation (LBIE) method for solving nonlinear problems, Computational Mechanics, 22, 174-186.

20. Zieniuk E. (2007), Modelling and effective modification of smooth boundary geometry in boundary problems using B-spline curves, Engineering with Computers, 23/1, 39-48.

21. Zieniuk E., Boltuć A. (2006), Bézier curves in the modeling of boundary geometry for $2 \mathrm{D}$ boundary problems defined by Helmholtz equation, Journal of Computational Acoustics, 14/3, 353-367.

22. Zieniuk E., Boltuć A. (2010), Iterative solution of linear and nonlinear boundary problems using PIES, Lecture Notes in Computer Science, 6067, 136-145.

23. Zienkiewicz O. (1977), The Finite Element Methods, McGraw-Hill, London. 